\title{
Arborescences
}

Revue d'études françaises

\section{Actes de cadrage, narratologie et herméneutique - à propos de l'indétermination énonciative dans Sujet Angot de Christine Angot}

\section{Liesbeth Korthals Altes}

Numéro 6, septembre 2016

Polyphonies : voix et valeurs du discours littéraire

URI : https://id.erudit.org/iderudit/1037506ar

DOI : https://doi.org/10.7202/1037506ar

Aller au sommaire du numéro

Éditeur(s)

Département d'études françaises, Université de Toronto

ISSN

1925-5357 (numérique)

Découvrir la revue

Citer cet article

Korthals Altes, L. (2016). Actes de cadrage, narratologie et herméneutique - à propos de l'indétermination énonciative dans Sujet Angot de Christine Angot. Arborescences, (6), 94-120. https://doi.org/10.7202/1037506ar
Résumé de l'article

Cet article présente une discussion théorique et méthodologique au sujet des façons dont nous faisons sens de brouillages des voix narratives et de cadrages génériques (autobiographie, biographie, fiction, autofiction) dans un texte littéraire. Le roman Sujet Angot de Christine Angot sert d'exemple afin d'illustrer la diversité des interprétations suscitées par les constructions narratives qui problématisent leur énonciation - et leurs valeurs. Attentive aux actes de cadrage ("framing » - Goffman) à l'oeuvre dans la lecture, l'approche métaherméneutique défendue dans cet article observe aussi les régimes de valeurs liés aux actes de classification (Boltanski et Thévenot) afin d'illustrer les bénéfices potentiels - pour l'éducation et pour la vie civile - d'une prise en compte réflexive des divers cadres conceptuels qui informent la lecture littéraire.
Tous droits réservés () Département d'études françaises, Université de Toronto, 2016 cécument est protege par la loi sur le droit d'auteur. L'utilisation des services d’Érudit (y compris la reproduction) est assujettie à sa politique d'utilisation que vous pouvez consulter en ligne. 


\section{SOMMAIRE}

Francis Langevin et Raphaël Baroni.

01 University of British Columbia et Université de Lausanne Introduction

Alain Rabatel. Université de Lyon 1

13 L'énonciation problématisante : en dialogue avec

Le Royaume d'Emmanuel Carrère

Émilie Goin. Université du Luxembourg

39 Analyse d'un discours d'action collective mis en récit.

L'Anarchie dans Les Cloches de Bâle d'Aragon

David Bélanger. Université du Québec à Montréal

54 En contre-jour : la représentation évanescente de l'écrivain dans le roman québécois contemporain

Raphaël Baroni. Université de Lausanne

72 Comment débusquer la voix d'un auteur dans sa fiction?

Une étude de quelques provocations de Michel Houellebecq

Liesbeth Korthals Altes. Rijksuniversiteit Groningen

94 Actes de cadrage, narratologie et herméneutique - à propos de I'indétermination énonciative dans Sujet Angot de Christine Angot

121

Marion Sauvaire et Érick Falardeau. Université Laval

Susciter le moment critique. De l'investissement de ressources axiologiques

Frank Wagner. Université Rennes 2

148 Quand le narrateur boit(e)... (Réflexions sur le narrateur non fiable et/ou indigne de confiance) 


\title{
Actes de cadrage, narratologie et herméneutique - à propos de l'indétermination énonciative dans Sujet Angot de Christine Angot
}

\author{
Liesbeth Korthals Altes. Rijksuniversiteit Groningen
}

\section{Résumé}

Cet article présente une discussion théorique et méthodologique au sujet des façons dont nous faisons sens de brouillages des voix narratives et de cadrages génériques (autobiographie, biographie, fiction, autofiction) dans un texte littéraire. Le roman Sujet Angot de Christine Angot sert d'exemple afin d'illustrer la diversité des interprétations suscitées par les constructions narratives qui problématisent leur énonciation - et leurs valeurs. Attentive aux actes de cadrage ("framing" - Goffman) à l'auvre dans la lecture, l'approche métaherméneutique défendue dans cet article observe aussi les régimes de valeurs liés aux actes de classification (Boltanski et Thévenot) afin d'illustrer les bénéfices potentiels - pour l'éducation et pour la vie civile - d'une prise en compte réflexive des divers cadres conceptuels qui informent la lecture littéraire.

Pour l'exploration théorique, méthodologique et interprétative de la question de la voix narrative qui sera au centre des réflexions qui suivent, l'œuvre de Christine Angot suscite des questions particulièrement intéressantes. La voix de l'auteure, tout d'abord, est incontournable, audible de manière parfois tapageuse dans le paysage littéraire et médiatique français, notamment depuis la publication de son septième livre, L'Inceste, en 1999 (son premier livre, $V u d u$ ciel, paru en 1990, avait eu une réception plutôt confidentielle). Cette voix s'entend à la fois dans un sens littéral, c'est-à-dire portée par la présence directe ou médiatisée de l'auteure dans ses nombreuses lectures et performances publiques ou ses interviews à la radio ou à la télévision ; mais elle se manifeste aussi souvent de manière plus oblique, c'està-dire comme objet de questionnement et de désir. Dans son œuvre littéraire, en tout cas, la mise en scène de la voix d'Angot joue sur toute une gamme d'effets qui se remettent en question réciproquement, oscillant en ricochet entre l'effet de pathos d'une personnalité privée — voix perçue comme authentique, émouvante ou au contraire comme intolérablement narcissique et casse-pied - et une polyphonie évasive, voire déroutante.

Prenons Sujet Angot, publié en 1998. Dans ce texte, l'ambiguïté des effets de voix pose un défi exemplaire non seulement à l'analyse et à l'interprétation, mais aussi, comme nous le verrons, aux théories et aux méthodes d'analyse de la voix narrative. Ce "roman", comme le veut la 
mention générique, met en scène un narrateur, Claude, qui, dans une longue lettre à son ex, Christine Angot, s'attache à définir leurs rapports de couple, maintenant défaits, et à cerner, fort de l'autorité que lui confère son intimité avec la personne privée et l'écrivaine débutante, la personnalité de Christine : "Toi. Qui tu es. Toi. Toi qui es unique. Je pourrai en parler des heures, moi » (p. 11). ${ }^{1}$ Mais qui parle, qui énonce ce propos sur Sujet Angot, dans ce texte farci d'insistants effets de réel et de tout aussi insistants excès de style? Et comment déterminer le genre de ce texte ? Cette dernière interrogation est intimement liée à la précédente, puisqu'elle contribue habituellement à cerner quelle voix, pour nous, au bout du compte, domine le jeu/je. S'agit-il d'un texte autobiographique, comme conventionnellement le titre et les nombreux détails (auto)biographiques corrects rapportés dans le texte le suggèrent? Christine/Angot a effectivement une fille, Léonore ; son ex s'appelle Claude ; de nombreux détails mentionnés ont été partagés en public par l'auteur ; etc. Or, si Christine Angot est certes au centre de l'expérience de lecture, c'est par ex - réel ou fictionnel - interposé, un dispositif retors, qui contamine l'effet de réel. ${ }^{2}$ Texte biographique, documentaire ? C'est Angot qui publie le texte, et non pas Claude : alloautobiographie, assumée par l'auteure ? "Roman ", comme le stipule la couverture, et donc peut-être fiction, ou seulement stylisation? La voie est toute tracée pour que puisse s'exercer sur la lecture littéraire un cadrage générique en vogue, celui de l'autofiction, genre qui fait de cette hésitation même son trait distinctif. Ce genre hybride problématise volontiers les conceptions établies concernant l'homogénéité de l'identité personnelle, l'écriture et la voix comme expressions du Moi ou encore le télescopage de l'auteur comme personne réelle, personnalité littéraire et figure qui se dégage de son œuvre. L'étiquette d'autofiction semble incontournable, avec ce dilemme générique plutôt lourdement aménagé, à la lumière, aussi, de ce que l'on peut savoir de l'auteure et de sa manière radicale et ambiguë de mettre en scène son propre vécu. Pourtant cette classification ne résout pas les problèmes de détermination du statut énonciatif et référentiel ou du poids rhétorique des voix mises en scène. Or le statut que nous accordons à la voix de Claude, et celle, en filigrane, de Christine - ou d'Angot - a des conséquences substantielles pour notre interprétation du texte, justement en ce qui concerne le programme littéraire formulé et performé, le rapport de la littérature au vécu, et son authenticité.

\footnotetext{
${ }^{1}$ Dans ce qui suit, le nom "Claude » désignera l'instance narratrice, et celui de "Christine », son ex-femme, l'écrivaine Christine Angot telle qu'elle appartient au monde narré, fictionnalisé/fictionnel, ou non. Par «Angot », en revanche, je réfèrerai à l'écrivaine réelle.

2 "La vie tout le temps, comme ça, infecte tout ce que je suis en train de faire ", écrit Angot dans L'Usage de la vie (1998 : 23). L'inverse est tout aussi vrai, sans doute, pour une part des lecteurs : la stylisation et l'artifice, une fois perçus, contaminent à leur tour les effets de "réel vécu ".
} 
Et pourtant, au risque de décevoir mon lecteur, et malgré l'intérêt du cas Angot, qu'on ne s'attende pas dans cet article à une analyse originale de ce roman. ${ }^{3}$ Sujet Angot servira uniquement d'illustration pour une discussion essentiellement théorique et méthodologique autour des questions suivantes : quelles sont les conséquences de ces ambiguïtés du cadrage générique (autobiographique, biographique, fictionnel, autofictionnel) pour les voix narratives que nous percevons dans ce texte et, plus généralement, dans un texte littéraire? Quel est l'impact de ces actes de cadrage pour la préséance de ces voix dans notre attention? La narratologie rend-elle suffisamment compte de la diversité des constructions de ces voix narratives, bien attestée dans les lectures véhémentes et contradictoires de critiques professionnels? Ces questions nourriront une réflexion qui ira de la narratologie à l'herméneutique, voire à la métaherméneutique. Par ce terme maladroit et un peu redondant, j'entends la reconstruction raisonnée des mécanismes et des conventions qui sous-tendent la manière dont nous interprétons les textes, y compris notre repérage des signes textuels.

Dans les pages qui suivent, sept thèses résument tout d'abord la perspective théorique proposée. J'enchaîne avec une discussion plus poussée de quelques concepts analytiques et de modèles développés ou, justement, insuffisamment explorés en narratologie, notamment : les notions de voix, de cadrage (Erving Goffman) et surtout de cadrage générique, puis l'idée de régimes de valeurs liés à des actes de classification (Luc Boltanski et Laurent Thévenot). Ces considérations sont illustrées par des références à des lectures possibles du texte d'Angot. En fin de compte, cette exploration voudrait nous faire réfléchir - nous, critiques littéraires, ou chercheurs dans ces domaines de la littérature et de la narratologie — aux cadres conceptuels, aux habitudes interprétatives et aux normes concernant la communication littéraire qui informent nos théories, nos méthodes et nos analyses littéraires. En épilogue figurent quelques réflexions sur la pertinence éducative, sociale et éthique du travail interprétatif et réflexif que peut susciter la littérature, ne serait-ce qu'à propos de la détermination des voix narratives.

\section{Sept thèses}

Voici donc, pour commencer, sept thèses qui résument l'essentiel du propos théorique en complément aux analyses narratologiques de la voix narrative. ${ }^{4}$

${ }^{3}$ Je me permets de renvoyer à mes analyses de ce texte d'Angot dans Korthals Altes 2014 : 199-204 et passim, et 2005.

${ }^{4}$ Ces thèses ne seront pas toutes approfondies dans cet article. Pour un développement plus poussé, voir Korthals Altes 2014. 
I. La détermination, par un lecteur, du statut des voix narratives ou des emboîtements énonciatifs/narratifs, avec leurs effets d'autorité, de dialogisme ou de polyphonie, dépend d'actes de cadrage interprétatifs de portée plus large. Je renvoie ici à la notion de frame (Goffman 1974); parmi ces actes de cadrage figurent en premier lieu la détermination du genre et de la situation énonciative/narrative qui prévaut, ainsi que la perception qu'a le lecteur du type de posture et de l'éthos de l'auteur (Meizoz 2007).

II. Ces actes de cadrage, qui sont pour ainsi dire constitutifs ou ontologiques puisqu'ils déterminent le "monde» et la scène énonciativecommunicative que le lecteur perçoit comme les plus pertinents, impliquent des horizons d'attente et des régimes de valeurs spécifiques concernant le type d'expérience dans lequel la lectrice pense s'engager ou, rétrospectivement, estime avoir été engagée.

III. L'analyse et la détermination de voix narratives ne reposent pas directement sur la description d'éléments textuels, mais relèvent d'une reconstruction (méta)herméneutique de ces actes de cadrage, entre autres générique, énonciatif, et postural, potentiels ou documentés.

IV. L'éventail des cadrages ontologiques qui se présentent à l'esprit du lecteur dépend, comme il a été souvent noté, d’indices génériques et d'indications de lecture (para)textuelles, mais aussi de l'expérience et de la compétence sociale et culturelle, littéraire et générale - du lecteur, comme de son habitus interprétatif (par exemple, psychologisant, moral, ou plutôt esthétique, etc.) et de sa disposition psychologique, notamment de sa capacité à l'empathie ou de son aptitude à gérer des dissonances cognitives. ${ }^{5}$

V. Le lecteur ressent souvent comme une richesse la possibilité de contextualiser le sens du texte de diverses manières, avec des contextes psychologiques, historiques, sociaux, philosophiques, éthiques ou autres, qui se superposent de manière pacifique. Mais quand il s'agit d'un texte qui met en scène des points de vue idéologiques ou éthiques controversés dans l'opinion, un cadrage générique et énonciatif incertain produit souvent, outre une angoisse classificatrice caractéristique, soit un plaisir intellectuel particulier, soit un rejet violent, processus qui semble apparenté au Gestalt switch décrit par Gombrich (1960 : 198) dans sa discussion de l'ambiguïté comme oscillation cognitive, ou au frame switch qu'implique la perception de l'humour ou de l'ironie (Koestler, 1989 : 35 ; commenté dans Korthals Altes, 2014: 224-226).

VI. Mieux comprendre la relation entre (1) ces cadrages du genre, de la posture d'auteur, et du type d'expérience proposée, (2) leur effet sur la détermination des niveaux narratifs, sur l'autorité et sur la crédibilité à accorder aux diverses voix narratives et (3) les régimes de valeurs qu'ils

${ }^{5}$ Cet aspect ne sera guère développé ici. Sur la notion de negative capability, voir par exemple Reuven Tsur 2006 : 8, 58. 
impliquent conventionnellement devrait permettre une meilleure saisie de la négociation sociale, interindividuelle, des significations et des valeurs à travers l'interprétation de textes littéraires.

VII. Si l'on pouvait établir que l'interprétation de la littérature occasionne une négociation de la valeur des valeurs, ceci justifierait l'exploitation plus systématique du potentiel réflexif, métacognitif, de l'interprétation littéraire (filmique, etc.) dans l'enseignement ou dans des ateliers de formation professionnelle ou personnelle pour adultes, par exemple.

Voyons de plus près quelques-unes des manières dont le phénomène désigné comme voix narrative a été conceptualisé, ou pourrait l'être.

\section{De quelques notions controversées, datées et pourtant pertinentes}

Il a été suffisamment noté que la notion de voix, telle qu'elle figure dans de nombreux travaux d'orientation narratologique, est peu homogène, peu rigoureuse et essentiellement métaphorique. ${ }^{6}$ Avec leurs ambitions de rigueur méthodologique et de portée générale, les approches narratologiques sont parfois étonnamment peu réflexives sur leur propre dimension interprétative et normative. Dans l'ensemble, la notion narratologique de la voix attire l'attention sur la manifestation discursive et l'agencement textuel de points de vue subjectifs sur le monde selon deux perspectives majeures : d'une part, l'analyse de la structure énonciative, avec son emboîtement de " niveaux " narratifs et les effets d'autorité, de mention, de mise en perspective ou autres manipulations liées à cette structure; d'autre part, l'analyse des voix d'un personnage, d'un narrateur et même d'un auteur, avec cette figure un peu paradoxale d'un auteur implicite/impliqué dont on chercherait à définir la voix : avec un peu de bonne volonté, on arrive à percevoir tout fragment de discours comme vecteur affectif, axiologique, idéologique et éthique, dimensions subsumées sous la notion de voix. Ces deux perspectives sont souvent combinées, en particulier en narratologie rhétorique (voir les travaux de James Phelan), ce qui tombe sous le sens si l'on souhaite comprendre comment la littérature offre une multiplicité de points de vue et organise des points de vue sur ces points de vue, modalisant la valeur des valeurs proposées.

${ }^{6}$ Voir par exemple l'excellent état de la question d'Els Jongeneel (2006) ou les deux numéros spéciaux de New Literary History 1998, vol. 29(3) et 2001, vol. 32 (3). 


\subsection{La narratologie, le narrateur et la voix}

Parmi les approches narratologiques, la poétique de la voix élaborée par Genette dans Figures III (1972) est sans doute la plus connue dans le monde francophone. Le Genette poéticien entendait faire contrepoids à une herméneutique subjectiviste et impressionniste avec ce modèle qui systématise les formes d'énonciation narrative, dégageant "ce qui se répète dans ce qui varie ", plutôt que "ce qui varie dans ce qui se répète ", pour paraphraser Frank Wagner (Wagner 2012 : \$9). Au cour de cette narratologie de la voix se trouve la figure du narrateur, ou plus précisément, « la façon dont se trouve impliquée dans le récit la narration elle-même, ou l'instance narrative : le narrateur et son destinataire" (1972: 234). Genette définit la notion de voix narrative à travers une analogie avec les modes verbaux (voix active/passive), dressant une typologie des niveaux narratifs qui permettrait d'analyser la fonctionnalité de la structure énonciative comme accès $\mathrm{au}(\mathrm{x})$ monde $(\mathrm{s}) \mathrm{du}$ récit, à l'information, à la focalisation, et à son rapport aux dimensions temporelles en jeu dans le récit. Mais, même chez Genette, il arrive que la notion de voix narrative glisse vers la manifestation d'une subjectivité, centre de perception et point de vue sur le monde (voir Jongeneel 2006 : 12). Son tableau, qui fait partie des connaissances narratologiques de base, distingue pour l'instance narrative quatre possibilités principales, selon leur niveau diégétique et leur rapport à l'histoire racontée (narrateur inclus ou non dans le monde de l'histoire), possibilités qui se laissent combiner: narrateur homodiégétique ou hétérodiégétique, d'une part, et narration extraxtradiégétique ou intradiégétique, d'autre part (Genette 1972 : 256).

Il a été reproché à Genette de construire sa typologie des niveaux narratifs sur une hiérarchie énonciative illusoirement stable et d'évacuer, côté rue, l'auteur comme autorité sur l'intentionnalité du texte, pour le faire rentrer côté jardin, incarné par un narrateur personnalisé (Aczel 1998 : 486). Son narrateur serait un vestige d'une vision du monde théologique, où la parole du plus haut aurait le monopole de la crédibilité et de l'autorité (Gibson 1996 : 145). Son modèle, en outre, ne permettrait guère l'analyse d'effets de voix indéterminées, ambiguës, ironiques ou autrement polyphoniques et occulterait le processus herméneutique sur lequel il repose reproche que je reprends dans cet article. Aczel observe ainsi, non sans raison, que " $[\mathrm{T}]$ o identify "who speaks", [...] it may be necessary first to identify how a particular voice speaks, and to distinguish it from other competing voices» (Aczel 1998 : 468), circularité qui n'est autre, dirais-je, que celle du cercle herméneutique. En fait, ce reproche vaut pour quasiment toutes ces approches qui rêvent d'exactitude méthodologique ou en font miroiter l'illusion, tout en reposant sur des actes interprétatifs et des normes communicationnelles et esthétiques insuffisamment reconnues, ou rapidement concédées, puis oubliées. À moins que l'on ne prenne le ductus 
taxinomique de Genette avec un grain de sel, comme le fait de manière convaincante Frank Wagner, et que l'on y voie non seulement une systématisation des conventions littéraires exprimées dans la forme narrative, mais autant de manières conventionnelles de théoriser et d'interpréter le texte narratif.

Reproches pertinents, qui n'empêchent pas le modèle genettien d'être utile, ne serait-ce que pour pointer là où ça dérape. Une narratologue, pressée d'analyser la narration dans ce texte déroutant d'Angot, par exemple, pourrait observer que, sur la base des premières pages du récit proprement dit, ce Claude est mis en scène comme un narrateur extrahomodiégétique. Or, le titre de Sujet Angot, outre les innombrables références à Christine Angot dans le texte, lui aura déjà mis la puce à l'oreille. Que ce nom propre d'Angot réfère à un personnage fictionnel ou à une personne réelle, notre narratologue chevronnée ne peut manquer au moins d'envisager que l'énonciation de Claude est sertie dans un niveau narratif supérieur, où c'est Christine (avatar fictionnalisé de l'auteure, ou même personnage fictif ?) ou Angot (coïncidence de la personne privée et de l'auteure ?) qui mène le jeu. Cette situation emboîtée de la voix du narrateur est d'ailleurs soulignée plus loin dans le texte par de brèves réponses dont la source n'est pas explicitée, du genre : «Tu comprends ce que je veux dire ? [Oui.] Tu trouves ça juste? [Oui.] L'écriture c'est exactement pareil »(p.120). Par inférence, notre narratologue attribuera sans doute ces interventions, qui suggèrent une intime connaissance mutuelle, à l'auteure Angot, ou à Christine, selon sa lecture plus ou moins personnalisante ou fictionnalisante. Claude suggère bien, en outre, qu'il a luimême offert à Christine le texte que nous lisons : "Cela me rend fort et fier, mon abandon dans ton écriture. [...] Christine veut s'en servir? Mais comment donc! [...] Vas-y, reine, vas-y magicienne» (p. 113-114). D’extradiégétique, cette voix narratrice devient intradiégétique. Ce narrateur si fort de son expertise concernant "sujet Angot " s'avère lui-même produit, au sens de montré, par Angot/Christine.

Dans la lignée de Genette, il est souvent postulé que «l'identification du statut du narrateur permet, indirectement, de dégager la finalité d'un récit " (Jouve 1997 : 26 ; je souligne, car c'est là que se cache la part d'interprétation de cette "identification»). Or, comment déterminer sur la base du modèle genettien le statut de ces voix et leur hiérarchie, voire leur effet de mise en perspective de l'énoncé ? Comment savoir à quelle voix se vouer, quels « je » sont en jeu, quelle vision du monde, quelle vérité est à prendre comme dominante dans la "finalité » ou l'intention du texte ? En effet, même si elle se laisse parfois aisément décrire par une argumentation, une hiérarchie énonciative formelle ne signifie pas automatiquement hiérarchie de la force d'appel rhétorique, ou hiérarchie des points de vue. La preuve, pour le voisin de bureau de notre narratologue, c'est manifestement la perspective de Claude qui mérite toute l'attention et la sympathie ; ce serait la "finalité du récit ", alors que pour la directrice du département, féministe pure et dure, ceci vaut 
justement pour la perspective d'Angot, la "vraie ", qui règle son compte à son ex avec ses armes à elle, dans cette querelle de couple où est aussi débattu quelque chose comme le droit d'auteur sur la fabrication de l'écrivain. Citons un seul passage en guise d'exemple :

Ce que tu écris en ce moment, ça ne va pas. Pardon. Mais les trucs
sexuels dont tu parles, les mecs, ce n'est pas toi ça. Tu n’y es pas. Tu
n'y es pas assez. C'est intellectuel, artificiel, en ce moment ce que tu
fais, excuse-moi. Et surtout : tu n'y es pas assez. Il faut que tu y sois.
Toi. Toi, Christine. Toi, Christine Angot, toi. Il faut que tu y sois, toi.
Pas seulement un ramassis de ce que les autres peuvent penser de toi.
Que tu as pris en note au café, au téléphone. Ça, ça ne fera jamais un
livre de toi. Ce qu'on peut penser ou dire de toi. Il faut que tu y sois,
toi. [... en plus tu fais un mauvais livre. (p. 10-11)

Claude se présente - ou est présenté se présentant - comme l'expert concernant l'écriture de Christine Angot ; c'est lui qui, depuis les premiers pas de Christine en littérature, détient le diapason qui fait résonner l'authenticité du style d'Angot. En même temps, le style de ce genre de passage, d'une répétitivité outrée, maniaque, risque de susciter la veine interprétative de maint lecteur, et miner l'attribution du discours à Claude (n'est-ce pas plutôt une Christine excédée qui mime le discours de son ex, ou une Angot qui, effectivement, ramasse les clichés sur son écriture à elle ?)

Bref, et je ne suis certainement pas la première à le constater, nous sommes loin ici d'une détermination formelle d'un type de narrateur et de ses effets de voix. Il ne s'agit pas d'une description de dimensions primairement textuelles, mais de l'articulation de facteurs pragmatiques qui déterminent notre accès aux signes textuels et à l'expérience offerte par un texte. Parmi ces facteurs figure de manière marquée le cadrage générique et énonciatif que nous adoptons, ce qui sera discuté plus amplement plus loin. Et dès que nous cherchons à déterminer comment concevoir ces voix narratives (comme personnage individualisé, comme " effet de sujet ", ou comme tissage de voix et de perspectives, personnalisées ou non) ou leur contribution aux effets idéologiques, éthiques ou autres du texte, voire leur rôle dans l'expression de l'intention de l'auteur (ou, selon une orthodoxie autonomiste, du " texte»), nous sommes sur le terrain de l'herméneutique. Ces remarques ne diminuent en aucune manière l'importance de l'appareil conceptuel développé par Genette. Elles ne font que souligner son statut quelque peu ambigu.

\subsection{La voix et la subjectivité dans le discours}

L'analyse de l'expression de la subjectivité dans le discours, qui est l'une des autres manières de concevoir la voix narrative, est quant à elle au 
centre des approches qui s'inspirent de la stylistique expressive de Bally ou, comme c'est aussi le cas chez Genette, de la linguistique du discours de Benveniste ou des théories pragmatiques (Kerbrat-Orecchioni 1980, Fludernik 1993). Arrêtons-nous au travail important de Monika Fludernik. Philologue de formation, Fludernik publie en 1993 un ouvrage de plus de cinq cents pages sur les langages de la fiction, qui dresse un inventaire impressionnant des expressions linguistiques de la subjectivité (sans toutefois se référer au travail similaire de Catherine Kerbrat-Orecchioni, qui ne lui était sans doute pas connu). Dans un deuxième ouvrage, qui a eu un grand retentissement dans les études narratologiques et littéraires, Towards a "Natural " Narratology (1996), Fludernik reconnaît plus explicitement que la base linguistique du repérage des voix narratives ne doit pas être surestimée, puisqu'une voix narrative est une construction du lecteur, "derived from implication and illocution" (Fludernik 1996 : 88). Sa définition de la voix narrative cherche dorénavant à intégrer dans son approche linguisticostylistique les apports de la pragmatique (communication quotidienne), de l'herméneutique et la phénoménologie (Ricœur), ainsi que ceux de la théorie cognitive des cadres cognitifs (on pense au travail de Marvin Minsky) et de la "folk psychology». La voix narrative devient alors "[t]he linguistically generated illusion of a voice factor, which can be defined empirically by a complex set of interrelated textual and contextual features and is corroborated by a mimetic reading of the text that stimulates this projection of a speaker or reflector function"(1996 : 344 ; je souligne). Cette définition complexe cherche à accommoder la tension, familière à beaucoup de chercheurs, entre l'exigence de rigueur méthodologique, manifestée dans l'intention de décrire la voix narrative comme un phénomène linguistique empirique (objectif) et la reconnaissance du rôle de l'interprétation (subjective). Mais la part de l'interprétation et des normes liée à la littérature continue à être sousproblématisée dans l'argument central de l'approche de Fludernik, qui consiste à affirmer que, dans notre lecture des textes littéraires, nous avons recours aux schémas cognitifs élaborés et mobilisés dans la vie quotidienne. Ainsi, Fludernik conçoit la voix narrative comme un "psychologically and morally coherent evaluating centre, usually constructed as a persona", tendance "naturelle " (Fludernik 1993 : 344) : la narration littéraire "invites a reading in terms of mimetic representation, and it is for this reason alone that certain stylistic textual effects are immediately recuperated or naturalised in terms of verisimilar 'voice', characters' perceptions and consciousness" (Fludernik 1993: 339). Or, s'il semble évident que notre compréhension primaire des textes littéraires (et non littéraires) requiert l'expérience rendue routinière et schématisée que nous avons pu accumuler dans la vie quotidienne, dans leur lecture de textes littéraires, de nombreux lecteurs font en sus appel à d'autres "schémas » et "scripts » cognitifs, de second ordre. Ceux-ci peuvent concerner les dispositifs énonciatifs dans lesquels un discours et ses particularités stylistiques ou thématiques sont pris, ou même la manière 
conventionnelle d'interpréter ce type de discours (effets d'ironie ou de provocation). L'enjeu de l'expérience littéraire ne réside pas nécessairement pour l'ensemble des lecteurs dans la reconnaissance de personnages « en chair et en os", sur la base d'une doxa psychologique et communicationnelle, comme nous le verrons un peu plus loin pour Sujet Angot. ${ }^{7}$ De plus, dans la vie quotidienne comme en littérature, différentes conceptions du Moi ou de la communication peuvent avoir cours, ouvertes à une compréhension plus feuilletée et complexe de la voix, de la parole et du « moi » individuels.

Fludernik se montre pourtant bel et bien sensible, par endroits, au fait que la littérature peut mettre en scène une expérience défamiliarisante du sujet et de la voix, notamment dans sa discussion des thèses provocatrices d'Ann Banfield. Pour Banfield, rappelons-le, dans la narration écrite, " [n]o one speaks [...], nor addresses anyone"; "subjectivity or the expressive function of narrative emerges free of communication » (Banfield 1982 : 97, 10). Seuls les personnages ou narrateurs s'exprimant en style direct à la première personne peuvent donc être considérés comme ayant/étant une voix ; les phrases narratives, elles, n'ont pas d'origine personnalisée et sont "linguistically free of the taint of subjectivity, of interpretation and evaluation" (Banfield 1982: 263). Du coup, une approche rhétorique perdrait une grande part de sa pertinence pour l'analyse de textes narratifs écrits. Fludernik, à juste titre, rétorque à Banfield qu' elle prend la notion de voix de manière trop littérale, trop grammaticale, et oublie que, justement, la langue fonctionne par allusions et inférences. Même dans une narration à "centre vide» ("empty center »), quantité de procédés expressifs nous amènent à imaginer une "implicit presence of a SELF in the text", "a perceiving consciousness", même s'il ne s'agit que d'une conscience " désincarnée " ("un-embodied») qui se résumerait à une "speaker function" (Fludernik 1993 : 391, 393). Cependant, peut-on ajouter, même une voix personnalisée n'est pas toujours à interpréter (uniquement) pour son poids de subjectivité. Un modèle théorique de la voix ou des voix narrative(s) devra tenir compte de ces différentes manières dont non seulement les textes et leurs auteurs, mais aussi les lecteurs, peuvent concevoir et susciter des effets textuels de subjectivité, les rapportant à leurs attentes normatives. Une remarque en passant: le recours aux sciences cognitives, aujourd'hui à la mode et que nous trouvons aussi chez Fludernik, nous rappelle la pertinence de l'herméneutique, qui, dans ses formes plus spécialisées, désigne l'importance d'une réflexion sur l'horizon d'attente et les préconnaissances (Gadamer), qui jouent un rôle crucial dans les processus interprétatifs, mais aussi, nous le voyons, dans les théories du récit littéraire.

La lecture de Sujet Angot semble bien donner raison à Fludernik, de prime abord: la plupart des lecteurs se sentiront sans doute

${ }^{7}$ Voir aussi, par exemple, le bel essai de Maurice Blanchot (1969) sur «Le "il”, le neutre». 
"immédiatement» incités à imaginer Claude et Christine en termes de "verisimilar 'voice', characters' perceptions and consciousness", y compris dans le cas de ces brèves interventions dialogiques dont l'énonciateur n'est pas spécifié. Quoi de plus reconnaissable que ce rapport de couple à la fois intime et féroce? Ce qui n'empêche qu'une lectrice plus sensible à ce que James Phelan a appelé la dimension synthétique des personnages - que j'étends ici aux voix narratives - pourrait concevoir de manière plus abstraite ces subjectivités évoquées $^{8}$ : non plus ce Claude ou cette Christine particularisés, mais des effet-sujets en quelque sorte dépersonnalisés, mis en composition de manière presque musicale, avec des effets de chœur (quand se dessine un "nous" non spécifié), de citation, de contrepoint et de polyphonie. Curieusement, même l'éthos d'authenticité peut persister dans une narration et une énonciation dont la source n'est ni individualisée ni déterminée. Une conception de la voix narrative rattachée nécessairement à une subjectivité distincte, voire à un personnage, rend insuffisamment compte de ce genre de possibilité.

Prenons à nouveau le cas de Sujet Angot. De nombreux lecteurs auront l'impression d'avoir un accès privilégié et authentique à Claude et à sa subjectivité "unique " à travers son propre éthos discursif, comme aussi à travers les préoccupations dont témoigne sa lettre. Au centre de ses réflexions figurent, outre son propre sentiment de déréliction après leur séparation, le style "unique » et la conception de l'écriture, mais aussi le comportement intime et public de Christine. Or l'écriture de Claude présente justement, et sur de nombreuses pages, les traits stylistiques et thématiques jugés typiques de l'écriture d'Angot, ou de Christine, comme nous le verrons dans une citation ci-dessous. Ceci vaut aussi pour la conception de l'écriture à laquelle Claude adhère et se conforme, avec son exigence du "poids de réel ». Claude lui-même ne manque pas d'attirer notre attention sur ces parallèles, lorsqu'il cite une critique qui commente l'écriture de Christine Angot en des termes qui s'appliqueraient tout aussi bien à son écriture à lui :

La phrase est cassée, délibérément, la continuité narrative absente. [...] [E]lle prolonge son entreprise autobiographique. Le quotidien est omniprésent, le "refus du récit » que revendique Angot, d'une extrême efficacité. (p. 84)

Quelle(s) voix et quel éthos discursif lisons-nous, du coup, dans son discours à lui ? Que devient la voix " unique " de Christine/Angot? Il en va du sérieux

8 Phelan (1989) a proposé de distinguer trois dimensions du personnage littéraire: mimétique (c'est-à-dire son effet-personnage, psychologique), thématique (le personnage comme représentant d'une époque, d'un milieu, d'un thème) et synthétique (ou métatextuel, quand le texte attire l'attention sur le personnage comme être de papier, artifice créé de toutes pièces). 
avec lequel on prendra les conceptions de la littérature et de l'écriture attribuées - et performées. Il en va aussi de la manière dont on voudrait juger de la qualité et de l'intérêt de ce livre : est-il à juger sur la base de sa capacité d'exprimer un $J e$ "vraisemblable" de manière authentique et prenante (selon des standards conventionnels, qui déterminent à quoi ressemble et « se sent " l'authenticité), ou de capter des effets-sujet multiples, fragmentaires et emmêlés, ou de sa virtuosité au jeu métafictionnel ? L'approche de la voix narrative proposée par Fludernik aura, me semble-t-il, tendance à suivre la voie d'une lecture et d'une soi-disant description narratologique mimétique, psychologisante, plutôt que de prendre pour objet, de manière plus analytique, la fabrication de ces effets de voix.

\subsection{La voix en narratologie rhétorique}

La notion de voix est également et évidemment centrale dans la narratologie rhétorique développée dans la lignée de Wayne C. Booth, qui vise à cerner au sein même de la structure narrative l'expression de points de vue multiples sur le réel et leurs effets de persuasion. Susan Lanser (1981), par exemple, conjugue brillamment cette narratologie rhétorique avec la théorie des actes du langage (voir aussi, par exemple, Kearns 1999). Lanser propose ainsi une grille heuristique détaillée pour analyser le "statut ", le "contact " (avec le lecteur ou d'autres publics) et l'attitude évaluative ("stance») du narrateur, visant en fin de compte l'auteur " extrafictionnel " à travers son texte. Avec cette visée sur l'auteur, Lanser se démarque nettement de Genette et de la doxa structuraliste, qui excluaient la figure de l'auteur de l'examen narratologique.

Plus récemment, dans la lignée rhétorique, Phelan inclut lui aussi sans hésitation non seulement l'auteur, mais aussi le lecteur dans le triangle lecteur-auteur-texte où se définit, selon lui, le sens du texte. En fait, l'approche narratologique dans laquelle il entend toujours s'inscrire abandonne alors ses prétentions - souvent implicites - d'analyse formelle et textuelle pour (re)devenir, toujours implicitement, protocole interprétatif, relevant dès lors d'une approche herméneutique. Cet "auteur ", chez Phelan reste suspendu entre auteur réel et auteur « impliqué », ce qui ne facilite pas la fonctionnalité de la notion (Phelan 2007, et passim; voir aussi l'étude fouillée de la notion d'auteur impliqué de Kindt et Müller 2006). La notion de lecteur, elle non plus, n'est pas vraiment théorisée et réfère généralement au chercheur lui-même, comme aux différentes lectures que celui - ou celle-ci peut imaginer.

Ces diverses narratologies rhétoriques tiennent pour évidente l'idée qu'un texte littéraire (narratif) est une forme de communication, de dialogue même, engageant le lecteur et l'auteur. De là qu'elles considèrent aussi

comme évidente la pertinence d'une perspective éthique. Une critique 
d'inspiration structuraliste pourrait interroger la part d'interprétation de cette méthodologie, sa subjectivité, voire son moralisme, en tout cas dans l'approche de Phelan. Une critique poststructuraliste, elle, pourrait viser l'humanisme " périmé » d'une approche qui perçoit des êtres vraisemblables, à la psychologie stable et cohérente, là où il n'y a que des effets textuels et une expérience de la discontinuité (Gibson 1996: 145 et passim). L'un des défis d'une théorie générale de la communication à travers la narration, littéraire et non littéraire, j'y insiste, c'est justement de rendre compte de la diversité des stratégies mobilisées pour faire signifier des structures narratives, au lieu d'en imposer une en particulier, qui correspondrait au parti pris du théoricien.

Et pourtant, quand on lit Sujet Angot, l'image du triangle auteurtexte-lecteur proposée par Phelan semble un correctif utile par rapport à l'approche structuraliste, axée sur le texte, au détriment de la part d'interprétation du lecteur ou du rôle que pourrait jouer l'image que le lecteur se fait de l'auteur. Cette approche narratologique rhétorique gagnerait toutefois à être articulée dans un cadre théorique explicite qui tiendrait compte des dimensions sémiotiques, historiques et sociologiques de la communication littéraire. Comme le disait déjà Booth, père de ces approches rhétoriques et communicationnelles de la fiction littéraire, l'herméneutique est le complément nécessaire et inévitable d'une approche rhétorique : dans notre analyse de la rhétorique d'un texte, nous accomplissons un travail d'interprète, déterminé par nos propres normes morales et esthétiques. Si toutefois on veut poursuivre l'ambition d'objectivation de la narratologie structuraliste, ce qui se défend, on pourrait par exemple chercher à cerner les mécanismes et les conventions qui sous-tendent ces pratiques interprétatives : ce serait un travail métaherméneutique. Dans cette visée, la narratologie, comme étude des procédés textuels mis en œuvre, devra collaborer avec, par exemple, l'histoire et la sociologie des conceptions de l'art et de la littérature, ou de l'artiste et de l'écrivain. L'attention pour la diversité des lectures, comme ici, la lecture de la voix narrative dans Sujet Angot, devrait être au cœur de cette approche. Il serait souhaitable de clarifier l'apport de ces diverses narratologies pour mieux comprendre le statut de ces instruments d'analyse.

\subsection{Polyphonie et métaherméneutique}

Même sommaire, cette revue critique ne saurait ignorer le travail de Mikhaïl Bakhtine, qui expose de manière convaincante la nature intertextuelle, citationnelle, sociale et plurielle des voix narratives à travers les notions de dialogisme, de polyphonie et d'hétéroglossie (Bakhtine 1970 [1929]). L'analyse bakhtinienne problématise la lecture personnalisante de la voix narrative, de même que la compréhension trop mécanique de l'idée, centrale aussi en analyse du discours, selon laquelle un discours caractérise son 
énonciateur à travers sa forme expressive même (voir par exemple Amossy 1999). Dans Sujet Angot, le discours apparemment monologique de Claude est déjà doublement orienté sur un destinataire : sur Christine, ce qui est souligné par les moments où le texte accueille les réponses (de Christine ?) aux questions de Claude et, au-delà, sur ses lecteurs virtuels et réels (cette manière de concevoir le texte transforme déjà l'énonciation, percevant la voix de Claude comme énoncée par un(e) autre). En outre, nous l'avons vu dans un court extrait, sa voix apparemment si personnelle est souvent minée par une exaspération stylistique ou encore traversée de discours étrangers (citation d'un texte biblique, d'un traité médical et autres corps intimement étrangers). Dès qu'une lectrice perçoit la possibilité d'une lecture polyphonique, comme cela risque d'être le cas pour la lettre de Claude, elle est entraînée dans un calcul d'intentions et d'éthos, faisant flèche de tout bois, de tout indice textuel, paratextuel et contextuel potentiel, calcul d'autant plus frénétique qu'il ne se laisse pas objectivement arrêter. Tout argument l'alimente.

L'idée de la pluralité inhérente à tout discours, que Bakhtine a bien mise en lumière, tombe sous le sens. Elle était pour Bakhtine fortement chargée de valeurs, liée à une profonde conviction démocratique, politique et éthique, dans un contexte historique hostile au pluralisme des idées. Radicalisée par la vague poststructuraliste, la notion a en quelque sorte servi de crédo, comme en témoigne l'expansion de la notion d'intertextualité chez Julia Kristeva. Pour moi, l'intérêt de cette approche de la voix réside aussi dans le fait qu'elle met en lumière l'impossibilité d'une approche simplement descriptive, ce qui souligne la nécessité d'une approche métaherméneutique qui reconstruirait les contextualisations et les raisonnements menant à la détermination, pour tel(s) interprète(s) particulier(s), de ces voix narratives ou de leurs effets de polyphonie. Il est vrai que Bakhtine lui-même s'est montré peu attentif, en tout cas pas systématiquement attentif au rôle que joue le lecteur dans l'appropriation d'un texte (ce qui justifie, par contraste, l'intérêt de l'herméneutique de Gadamer ; voir sur ce point Jongeneel 2006 : 20-23).

Ce n'est sans doute pas un hasard si la notion de voix en théorie narrative reste hétérogène et controversée. Au-delà de ses fonctions analytiques, le concept est souvent investi de fonctions idéologiques, voire éthiques. Les appareils analytiques proposés s'avèrent habités par ce que j'appelle des programmes herméneutiques qui relèvent de conceptions normatives diverses. À propos de la littérature (communication/dialogue, ou non ?) et son rapport au réel, par exemple ; concernant la communication et l'interaction avec le lecteur, ainsi que le rôle et la posture de l'auteur : tel théoricien concevant ces voix comme, idéalement, personnalisées ; ou enfin, autre programme herméneutique, l'expérience littéraire comme dialogue avec un auteur perçu comme réel alors que tel autre lecteur se représentera ces voix narratives de manière moins personnalisée ou homogène et maintiendra fermement l'auteur hors de l'analyse. Une narratologie qui se veut générale devrait considérer ces pratiques interprétatives et ces programmes 
herméneutiques, implicites ou explicites, comme faisant partie de l'objet à analyser au lieu de se ranger sous la bannière de l'une d'elles. Elle devrait aussi abandonner la prétention de simplement décrire des phénomènes textuels et considérer la voix narrative plutôt comme la construction interprétative d'un lecteur, où les dimensions textuelles, stylistiques et énonciatives prennent leur pertinence comme indices sémiotiques dans le cadre de conventions interprétatives diverses. Différents aspects du matériau textuel se donnent alors à lire comme indices, par différents lecteurs, sur la base de leurs actes de cadrage primaires. Les mêmes éléments textuels peuvent dès lors être interprétés différemment - on ne le sait que trop bien. Ceci ne veut pas dire pour autant que le narratologue soit incapable de généralisations et en soit réduit à constater la subjectivité incontournable des lectures. Dans la démonstration de la pertinence de ma lecture, l'instrumentaire narratologique est utile d'une part comme heuristique : les notions proposées par Genette, Fludernik, Phelan ou Bakhtine attirent notre attention sur des aspects précis du potentiel sémiotique d'un texte. ${ }^{9}$ D'autre part, dans une perspective rétrospective et analytique, ces notions offrent un vocabulaire pour formuler notre perception de ces éléments formels du texte qui font signe pour nous et qui marquent les limites des approches plus essentiellement thématiques et mimétiques.

\section{Actes de cadrage}

Dans ce qui suit, je voudrais examiner quelques notions qui permettent, je pense, de reformuler certaines des questions qui précèdent et de rendre compte, au moins en partie, de notre accès au texte et des mécanismes qui sous-tendent la diversité des lectures. L'accent portera en particulier sur certains actes de cadrage, qui semblent fortement déterminer les pistes de lecture adoptées. Il est clair que ces considérations métaherméneutiques gagneraient à être testées et affinées dans l'examen empirique de comportements de lecteurs réels. Pour l'instant, plus modestement, je souhaite formuler des réflexions susceptibles de faire émerger des hypothèses qui donneraient lieu à des analyses historiques, sociologiques et autres, incluant un examen empirique. Pour cette réflexion sur la détermination des voix narratives, la notion de cadrage joue à mon sens un rôle central.

\footnotetext{
${ }^{9}$ Voir Kindt et Müller 2003, qui proposent une analyse de la narratologie et de son rapport à l'interprétation proche de la mienne, insistant au bout du compte sur l'utilité heuristique de l'appareil conceptuel narratologique.
} 


\subsection{Cadrage}

Erving Goffman, sociologue de la vie quotidienne, explique dans son ouvrage Frame analysis. An Essay on the Organization of Experience (traduit en français sous le titre Les Cadres de l'expérience) que nous faisons sens de nos expériences et des situations auxquelles nous nous trouvons confrontés grâce à notre capacité mentale de cadrage (Goffman emploie les termes de frames et de framing). Cette idée n'était certes pas neuve : Goffman lui-même s'inspirait du travail de l'anthropologue Gregory Bateson. Mais il en montre de manière convaincante la force analytique. Ces actes de cadrage sont des «schemata of interpretation ", qui permettent à des individus ou des groupes " to locate, perceive, identify, and label events and occurrences, thus rendering meaning, organizing experiences, and guiding actions " (Goffman 1974, 21). ${ }^{10}$ L'importance de cette compétence classificatrice est soulignée par les quiproquos, les impostures et les dilemmes de cadrage qui prolifèrent dans la vie quotidienne, comme dans nos représentations artistiques (le théâtre est un lieu de démonstration privilégié chez Goffman), religieuses, mythologiques ou politiques, avec les hésitations et les erreurs qui s'ensuivent concernant l'attitude interprétative et évaluative et le cours d'action à adopter. D'où l'importance, également, des indices ou "clés de cadrage " ( framing keys »), qui sont des signaux conventionnels indiquant le "mode " selon lequel une situation doit être comprise (Goffman 1974, 43-44). Selon la clé de cadrage que nous choisissons, une affirmation sérieuse bascule en ironie, une rixe dans la rue devient performance théâtrale. Mais ces clés de cadrage sont ellesmêmes des signes que nous sélectionnons et percevons sur la base de... cadrages préalables, selon une circularité herméneutique inévitable.

La notion de cadrage semble pertinente pour analyser les phénomènes de voix narrative en jeu dans cet article. ${ }^{11}$ On peut penser ici aux clés de cadrage que sont l'éthos préalable d'un auteur, qui se dégage de son œuvre déjà publiée ou de sa posture perçue (Meizoz 2007) : l'image d'Angot que s'est formée le lecteur - comme auteure " authentique » et "rebelle », comme "Jeanne d'Arc» ou "Bécassine des lettres», comme auteure

${ }^{10}$ Il faut se garder de confondre cette notion goffmanienne avec la notion de "frame " proposée en théorie cognitive (voir Minsky 1977). Cependant, comme l'observe Werner Wolf, ces différentes approches en termes de "frames", qu'elles soient goffmanienne ou cognitivistes, convergent "in one frame function, namely to guide and even to enable interpretation. [...] [O]ne can argue that frames as "keys" to interpretation are at least "metaconcepts": concepts that regulate the application of other concepts " (Wolf 2006, 3-4).

${ }^{11}$ Voir l'approche convergente de Raphaël Baroni. Dans son article "The Relevance of Irrelevance in Intriguing Narratives ", par exemple, il met en relation les actes de cadrage opérés par un lecteur avec sa façon de déterminer la pertinence d'une énonciation : "We see that the same text can be considered as relevant or irrelevant according to the frame we use to interpret it » (Baroni, à paraître) ; pour la notion de pertinence, voir Sperber et Wilson 1995 [1986]. 
"métafictionnelle» - risque de l'orienter vers telle interprétation du texte : écriture qui clame l'expérience vécue au nom de la vérité, ou qui démonte et démasque ce désir tout en le performant, par exemple. Ce cadrage construit par l'éthos préalable de l'auteure pourrait mener la lecture vers telle perception des voix de Claude, de Christine, voire de l'auteure. Le cadrage du type d'auteur est intimement lié au cadrage générique du texte. Ensemble, ils sont lourds de conséquences, car ils déterminent le rapport qu'un texte est considéré entretenir avec le réel, avec son auteur en tant que personne privée, et donc de l'expérience de lecture suscitée. Pour des textes publiés comme authentiquement autobiographiques, par exemple ceux de Binjamin Wilkomirski ou de James Frey, l'éthos publiquement manifesté de l'auteur fonctionne comme garantie du rapport du texte au réel postulé, consacré dans une classification dans un des genres de l'écriture du réel, autobiographie ou documentaire. Ces textes ont été plus tard "démasqués " comme fictions, et nombre de lecteurs, y compris professionnels, considèrent que ces cas ne relèvent pas de la liberté de la fiction, mais constituent une imposture, justifiable d'un jugement moral (nous verrons plus loin le lien entre la classification dans un monde particulier et l'application d'un régime de valeurs spécifique). Philippe Lejeune ne parle pas par hasard de pacte autobiographique. Tout genre de discours, on le sait, a une dimension contractuelle, pragmatique, qui détermine notre manière de "prendre » et de comprendre le texte.

Dans un cas comme celui de Sujet Angot, les clés de cadrage générique sont assez nettement contradictoires. Ceci n'empêche pas différents critiques, et sans doute nombre de lecteurs, de se sentir déroutés, voire manipulés par l'indétermination générique, ce qui se traduit en incertitude concernant le sens, la valeur (littéraire, mais aussi morale) du projet d'écriture d'Angot: cette écriture est-elle vouée à clamer et ressasser le vécu ? Ou est-ce qu'elle thématise et interroge justement cette insistante expression du réel vécu et cette soif d'authenticité largement diffusées dans les médias? Les thèmes développés dans les textes d'Angot sont chargés d'affects : ici, la rupture du couple, le sentiment de déréliction du partenaire; ailleurs, l'inceste, par exemple. Les voix narratives expriment leur expérience existentielle avec de forts effets de pathos, accompagnés des topoï d'un éthos d'authenticité (écriture non policée, proche du discours oral spontané, ponctuation émotive...). Effets qui sollicitent une attitude de lecture empathique, psychologisante et morale, et qui peuvent suggérer une esthétique de l'immédiateté. Or les dissonances dans cet appel rhétorique sont tout aussi nombreuses. En fin de compte, comme j'ai eu l'occasion d'en faire la démonstration ailleurs, l'écriture d'Angot - souvent perçue comme la voix de l'auteure - bloque l'intimité et l'immédiateté qui semblent proposées (Korthals Altes 2005 et 2014). Est-ce là justement une manière de garantir l'authenticité de son écriture? Ou s'agit-il d'une structure complexe, contradictoire et stratégique, frauduleuse selon certains, pour capter et 
maintenir l'attention du lecteur, et "vendre" ? Authenticity sells, mais l'authenticité performée et problématisée confond les effets de scandale et d'exhibitionnisme avec des valeurs littéraires et morales établies, de recherche et d'exploration. La détermination du genre du texte est, pour le lecteur, une façon de répondre à ces questions en recourant à son propre rapport au réel, au «vrai » et à l'auteure.

\subsection{Scénographies et cadrages}

La distinction que fait Dominique Maingueneau entre scène englobante, scène générique et scénographie prend elle aussi tout son sens quand on la lit dans une perspective goffmanienne. Elle systématise, pourraiton dire, un emboîtement de cadrages constitutifs pour l'expérience de lecture littéraire (mais le phénomène se produit dans n'importe quelle sorte de communication, puisqu'on peut toujours jouer avec différents cadrages génériques et communicationnels, ou brouiller intentionnellement les clés de cadrage). La scène englobante, on le sait, permet d'abord de catégoriser le statut pragmatique d'un texte comme fiction ou non-fiction, et comme appartenant au type de texte littéraire, religieux, philosophique, technique ou autre. Le cadrage générique ou la scène générique retenue par le lecteur suscitent des attentes conventionnellement rattachées à un choix de (sous-) genre. La scénographie d'un texte, enfin, correspond à une situation de communication concrète, évoquant par exemple celle d'une confession, d'un dialogue, ou d'un rapport de police (Maingueneau 2004 : 103-107, 190202). J'ajouterais que ces divers cadrages de la situation narrative soumise à notre attention peuvent impliquer à chaque niveau des attentes spécifiques concernant le rapport à la réalité (fidélité référentielle requise, ou non) et concernant la pertinence même des conjectures à propos d'un auteur, de sa voix, de son éthos et de son positionnement idéologique ou autre. Ainsi, les attentes concernant la fiabilité, l'authenticité ou la sincérité de l'engagement d'un auteur par rapport aux points de vue exprimés par un narrateur ou un personnage sont en général nettement plus fortes dans les genres qui se rapprochent du régime non fictionnel, tels que l'autobiographie sous ses diverses formes, le roman engagé, le roman d'idées ou la docu-fiction. Dans ces genres, le positionnement idéologique ou autre au niveau de la scénographie est censé être en continuité avec celui du texte au niveau englobant et au-delà, jusqu'à la posture et à l'éthos de l'auteur, attestés dans les actes.

L'emboîtement de cadrages que représente le modèle de Maingueneau ne prédit cependant pas la force d'appel rhétorique et idéologique des différents niveaux impliqués, ainsi qu'en témoigne la réception d'œuvres de Christine Angot, de Michel Houellebecq et de nombreux autres auteurs qui jouent sur l'ambiguïté d'une énonciation souvent provocatrice. Une 
scénographie d'apparence autobiographique ou documentaire peut déployer de manière si suggestive les indices et les topoï d'un éthos non fictionnel que ce cadrage de l'éthos de l'énonciateur (souvent amalgamé à l'auteur) triomphe aisément des signalisations d'une scène englobante fictionnelle, qui, elle, inviterait à détacher l'œuvre de l'auteur comme personne privée. Ces phénomènes de contagion de cadrage générique seraient à étudier avec précision, en comparant, par exemple, les habitudes de lecture mimétiques esthétiques.

Dans ma lecture de Sujet Angot, par exemple, je pourrais me laisser immerger dans la scénographie, puissante dans ses effets de réalisme, que constitue la lettre de Claude. Je pourrais ressentir le pathos du discours de Claude comme authentique et émouvant, en dépit - ou justement en raison - de son évidente mauvaise foi quand il parle de son ex, qui font de lui un narrateur non fiable. J'aurais tendance à étendre (un peu vite, mais l'émotion faisant son œuvre) à cette scénographie les indices génériques d'autobiographie, proposés par le titre et les références à ce que l'on sait de la vie d'Angot. Je lirais alors un livre sur Lui à propos d'Elle, que je percevrais comme vrai, authentique, en une lecture "mimétique", comme dirait Fludernik. Je trouverais normal de jauger la teneur morale du comportement de Claude, y compris son écriture et sa manière de faire le portrait de Christine.

Mais je pourrais aussi être attentive de prime abord à la signalisation contradictoire de la scène englobante, où se définit le statut du texte. Résoudre la question de la scène englobante par un cadrage en autofiction n'aide pas beaucoup, puisque l'autofiction se laisse tirer vers l'autobiographie comme vers la métafiction. Dans le premier cas, l'intérêt du texte demeure existentiel, psychologique: je lirais alors un livre sur Christine, ou Angot à propos de [Lui sur Elle]. Dans cette lecture, l'arroseur se trouve arrosé, puisque ce Claude qui prétend connaître Christine sous toutes ses coutures est à son tour mis à nu dans sa propre manière d'écrire. Si mon cadrage tendait vers la métafiction, je lirais par exemple un livre d'Angot sur [une mise en scène de [Lui sur Elle]]. L'intérêt de ma lecture pourrait résider dans le plaisir que je dériverais du jeu avec les attentes génériques, thématiques, émotionnelles ou morales suscitées. Les thèmes qui me retiendraient seraient non pas tant ces personnages particularisés, avec leur expérience existentielle ou leur référentialité réelle, mais le caractère évasif du Moi, jusque dans l'autoréflexion écrite; ou la prise de conscience du désir du lecteur - moimême - de connaître intimement l'auteure à travers sa voix, tendance nourrie par les interminables rencontres-avec-l'écrivain, voix que nous croyons déjà connaître, et que nous traquons jusque dans l'éthos discursif de son narrateur, son métissage interdiscursif et le dispositif énonciatif même. Ce désir du lecteur se conjugue ici, dans un mélange de cadres troublant, avec le désir d'une auteure de se connaître elle-même, et celui, érotique, affectif et littéraire de l'ex-mari : dispositif où la voix de l'autre parlant de Moi ne restitue pas à 
ce Moi une essence, mais au moins constitue ce Sujet Angot en objet d'attention et démontre - par le creux qu'il laisse - qu'il a été aimé. Mais connaître l'autre prend aussi, dans ces effets de miroir, toutes les connotations d'emprise violente que décelait Emmanuel Levinas, pour qui "[p]osséder, connaître, saisir sont des synonymes du pouvoir»(Levinas 1983 : 83). Selon notre cadrage générique, notre horizon interprétatif et évaluatif change et, avec lui, même les effets d'ironie auxquels on risque d'être sensible : non plus l'ironie visant les comportements ou les points de vue de Claude, ou de Christine/Angot, mais celle qui expose ce désir du public pour un auteur, notre propre soif d'authenticité, nos manières de lire.

Les textes perçus comme littéraires exploitent volontiers, on le sait, les tensions entre cadrages alternatifs, multipliant des indices génériques contradictoires à ces niveaux pour ainsi dire constitutifs ou ontologiques. L'incertitude par rapport au cadrage énonciatif et générique, mais aussi par rapport à l'éthos et au "type "d'auteur auquel on pense avoir affaire, devient incertitude concernant la stratégie interprétative et le régime de valeurs indiqués, ce qui exige un travail interprétatif plus intensif.

\subsection{Cadrages et régimes de valeur}

Les hésitations et conflits de cadrage suscités par des œuvres ou des auteurs littéraires offrent un riche matériau pour une analyse qui s'intéresse aux (courts-circuits des) raisonnements selon lesquels professionnels de la littérature ou simples lecteurs construisent l'univers textuel et son sens, perçoivent des points de vue sur le monde et en arrivent à des jugements de valeur. C'est là, enfin, que réside pour moi l'intérêt du travail de Luc Boltanski et de Laurent Thévenot qui, dans De la justification (1991), rattachent à un type d'argumentation et à un régime de valeurs distincts la manière dont nous classifions une activité ou un discours dans un " monde " particulier.

Boltanski et Thévenot, en effet, distinguent globalement dans la société française des années 1980 six domaines qu'ils nomment "Cités » ou "Mondes" (je retiendrai ce dernier terme) : le monde inspiré, avec les arts comme activité exemplaire, et des valeurs caractéristiques telles que la créativité et l'originalité ; le monde domestique, exemplifié par la vie familiale, avec des valeurs comme le respect de la tradition, l'amour et le soutien mutuels; le monde de l'opinion, avec les médias pour exemple, et la renommée comme valeur-standard; le monde civique, représenté par la politique ou le système juridique, avec des valeurs comme la responsabilité, la justice ou le bien commun; le monde industriel, qui englobe les divers cercles du travail, avec des valeurs comme l'efficacité, l'application et la compétence technique ; finalement, le monde du commerce, avec des valeurs comme la rentabilité et l'efficacité (Boltanski et Thévenot 1991: 201-260). Ces mondes ne 
correspondent pas à des domaines sociologiques clairement définis, ni à ce que Pierre Bourdieu a décrit comme champs, mais, dans un esprit plus goffmanien, à des manières basiques de classifier nos activités, empruntées à notre expérience quotidienne. Ces classifications souvent intuitives et non conscientes nous permettent de nous rapporter à de nouvelles situations en leur associant des régimes de valeurs conventionnellement attachés à ces mondes. Par exemple, parler d'une Faculté de Lettres comme d'une famille appelle un régime de valeurs "domestique" (soutien mutuel, responsabilité pour le groupe, par exemple); proposer de diriger cette Faculté comme une entreprise suscite un régime de valeurs industriel et commercial (rentabilité, efficacité) ; décrire la Faculté comme une communauté où sont formés les cadres d'une nation la catégorise dans un monde "civique", avec comme valeur directrice, celle de responsabilité civique.

L'identification et la détermination des mondes et des régimes de valeurs pertinents s'avèrent importantes pour les lecteurs qui cherchent à savoir comment faire sens d'un texte littéraire. Dans le cas de Sujet Angot, la scénographie proposée dès la première page - la lettre de Claude à Christine Angot - invite aisément au classement de ce texte dans la catégorie du monde «domestique». Ce cadrage suscite des attentes concernant l'éthos du narrateur-personnage. Sa sincérité et sa fiabilité feront évidemment l'objet de notre attention, dès lors qu'elles font appel à des normes morales largement répandues au quotidien. Même si ma lecture est attentive au dispositif énonciatif dans lequel la lettre de Claude est citée, ce régime de valeurs "domestique" ne dément pas. Il m'amènerait alors à mettre en doute la fiabilité de la narratrice Christine, ou Angot, que je construis comme l'origine de cet emboîtement ou mise en boîte énonciative. Si, par exemple, je faisais correspondre ces personnages de "roman" à des êtres réels, je pourrais reprocher à l'auteure une exploitation et une exhibition immorales de l'expérience de son ex-mari, classant du coup le texte aussi dans le monde " civique", avec son régime de valeurs propre. Si je lis le texte à travers un cadrage générique autofictionnel, en revanche, cela revient - du moins dans une certaine mesure, étant donné l'ambiguïté de la classification même - à le soumettre au régime de valeurs du monde "inspiré », avec l'ambiguïté, la polyphonie, l'épaisseur des couches de sens comme valeurs déterminantes, prévalant sur l'horizon moral et psychologique.

Certes, le modèle de Boltanski et Thévenot ne pèche pas par excès de rigueur. Leur notion de "monde " et les régimes de valeur qu'ils y rattachent sont décrits de manière plutôt ad hoc. Mais leur parti pris presque antithéorique et anti-méthodique reconnaît avec une rafraîchissante lucidité la part de reconstruction herméneutique et d'analyse raisonnée à l'œuvre dans les stratégies de justification et de négociation de cadrages, avec leurs jugements de valeur afférents. Leur modèle d'analyse possède clairement une valeur heuristique et paraît fructueux, en particulier, pour analyser des auteurs controversés et des œuvres perçues comme hybrides, suscitant des dilemmes 
de cadrage. Cette approche a l'avantage d'attirer l'attention sur les types de valeurs - leur hétérogénéité et souvent leur entremêlement - en jeu dans la circulation des œuvres littéraires, ouvrant ainsi à une image plus dynamique et plus réaliste que celle d'un "champ littéraire" tendu entre un pôle autonome et un pôle hétéronome.

Le type d'analyse de la voix que je voudrais proposer ici revient à reconstruire, sur un mode métaherméneutique, les actes de cadrage accomplis par des lecteurs réels (y compris nous-même) ou virtuels, qui entrent en jeu quand nous distinguons telles voix et telles structures narratives, à travers des cadrages qui sont aussi des classifications de l'univers narratif dans un monde particulier (au sens de Boltanski et Thévenot), avec leurs régimes de valeur particuliers. Articulé à une étude des actes de cadrage (générique, auctorial) qui contribuent à déterminer nos accès au texte et notre attitude de lecture, ce genre d'analyse permet de mieux comprendre et de décortiquer la réception mouvementée de textes comme celui d'Angot. Réception toujours polémique, voire parfois véhémente, en effet, puisqu'elle met en jeu non seulement des valeurs, mais aussi la nécessité de garder clairs et distincts des régimes de classification dans une réalité souvent complexe et opaque. La validité de ce genre d'analyse, cependant, n'est pas garantie par des preuves textuelles objectives, mais dépend plutôt de la qualité du raisonnement.

\section{Littérature, interprétation et métacognition}

L'indétermination énonciative ou générique n'est certainement pas réservée à l'expérience littéraire, comme l'a souligné Goffman avec brio. Le cadrage correct des promesses d'un ami ou d'un partenaire d'entreprise inclut une estimation adéquate de leur éthos et du statut générique et énonciatif de leur discours. Il est crucial de savoir développer une compétence de cadrage, avec toute la subtilité et la flexibilité requises, puisque cela entraîne des conséquences concrètes : ce cadrage détermine comment nous pensons devoir agir, même si dans le régime de l'art, quel que soit le désir d'intervention dans le réel que nourrit un auteur, le lien avec la nécessité d'agir est en apparence moins direct.

L'approche proposée abandonne l'ambition à mon sens illusoire de formuler une méthode descriptive objective, indépendante de l'interprétation, des phénomènes de voix dans des textes particuliers. À la lumière de ce que l'on comprend des mécanismes de la production du sens et des valeurs grâce à la sociologie, à la sémiotique et aux sciences cognitives, il semble plus prometteur et judicieux de s'attacher à reconstituer les schémas, en grande partie conventionnels, selon lesquels des lecteurs font sens à partir des textes. Cette recherche peut mener, dans une perspective historique et sociologique, à reconstituer les conceptions de la littérature qui sous-tendent les habitus interprétatifs au-delà de leurs conditionnements sociologiques et 
psychologiques, ce qu'illustrent bien les travaux d'Alain Viala, de Paul Bénichou, de Gisèle Sapiro, de Nathalie Heinich et d'autres. Cette approche a aussi une portée heuristique pour analyser les débats et controverses autour d'œuvres polémiques et de leurs auteurs, en attirant l'attention sur les opérations de classification et les régimes de valeurs qui entrent en compétition.

On peut aussi s'interroger, de façon plus générale, sur la fonction de l'intense activité imaginative et interprétative dont doivent faire preuve les lecteurs, lorsqu'ils sont confrontés à ces œuvres qui suscitent des dilemmes de cadrage. Rappelons ici que nombre de théories anthropologiques, philosophiques, psychologiques ou autres soulignent la fonction métacognitive des arts, comme aussi de la narration. Dans sa théorie cognitive de la culture, Merlin Donald, par exemple, décrit les arts et la narration comme des instruments grâce auxquels une culture organise la métacognition, elle-même nécessaire pour maintenir la flexibilité dans l'adaptation à de nouvelles situations (Donald 2006). L'intérêt du roman ne réside pas uniquement dans les mondes et les points de vue sur ces mondes qu'il évoque, mais aussi dans l'intensification et la re-présentation des opérations mentales à travers lesquelles nous faisons sens de notre expérience et attribuons des valeurs. La fiction littéraire en appelle à notre capacité à nous représenter des mondes et des expériences subjectives de ces mondes, des points de vue sur ces expériences, puis à nous rapporter nous-mêmes à ces points de vue sur cette expérience. Elle exerce notre capacité à jongler mentalement avec différents niveaux de réalité et à jauger l'autorité, la fiabilité et la valeur des points de vue sur des points de vue et des valeurs. Le caractère lourdement redondant de toutes ces formulations exprime le caractère récursif de la métacognition à laquelle donne lieu l'expérience de la littérature. Pour complexifier encore le processus : lorsque nous faisons l'effort de formuler une interprétation d'un texte un tant soit peu complexe, et de confronter cette interprétation à celles des autres, la métacognition s'engage encore plus loin dans la spirale. L'interprétation de textes complexes, surtout comme activité sociale, est en soi un entraînement à la gestion de la complexité, de l'ambiguïté et de la dissonance cognitive. Pour certains, il en résulte ce plaisir particulier pris à la dextérité dans l'exercice de nos capacités physiques ou mentales, que le psychologue Karl Bühler appelait Funktionslust (1965 : 157). Pour d'autres, les incertitudes interprétatives induites par une œuvre d'art sont source d'irritation, de désarroi et de rejet. Il serait intéressant d'explorer dans une approche qui combinerait métaherméneutique et recherche empirique comment des lecteurs concrets perçoivent les brouillages de cadre et les incertitudes qui en résultent : quelles sont leurs stratégies pour y faire face et faire sens du texte, et quels rôles jouent dans ce processus les aspects textuels et paratextuels, l'expérience et la compétence littéraires, ainsi que des aspects spécifiques d'une personnalité (notamment la capacité à l'empathie, au perspective-taking, à la gestion des dissonances cognitives) ? Je propose 
l'hypothèse qu'en cas de brouillage ou de dilemme de cadrage générique et énonciatif, le lecteur se réfère, entre autres, à l'éthos et à la posture qu'il pense pouvoir attribuer à l'auteur. ${ }^{12}$

\section{En guise d'épilogue}

Pour terminer sur une note informelle et personnelle, qui met en jeu mes propres conceptions normatives de la société, de l'interaction et de la réflexivité que rend possibles l'expérience artistique, je suggérerais qu'une démocratie requiert de ses citoyens un degré élevé de cette compétence à gérer la complexité, à l'analyser et à formuler un jugement, au-delà de l'empathie empathie qui attire tant l'attention actuellement. Dans cette perspective, outre son intérêt théorique et universitaire, l'approche métaherméneutique se prête à une exploitation pratique, sociale et éthique dans des contextes éducatifs ou des entraînements socioprofessionnels, par exemple. Il m'est ainsi arrivé de diriger des groupes de travaux pratiques centrés sur l'interprétation d'un texte littéraire (souvent un récit bref, comme L'Hôte, de Camus) avec des groupes composés par exemple de chefs d'entreprise, de professeurs du secondaire ou de lycéens. En voici une rapide impression, non théorisée : les participants, dont le nombre ne dépasse idéalement pas douze, lisent le texte avant la séance. Au début de notre séance - qui dure environ trois heures pour les adultes, une heure pour les adolescents -, les participants partent se promener à deux, en partageant leur expérience de lecture, avec en main quelques questions pour les mettre en route. De retour après dix minutes, ils résument pour le groupe la position de leur interlocuteur sur un certain nombre de points précis concernant le texte (points thématiques, formels et contextuels, mais aussi concernant ce qui leur importe dans l'expérience de la lecture littéraire). Suit une discussion, d'abord sur l'interprétation du texte, puis sur nos différentes manières de lire, de faire sens, d'évaluer les positions de valeur que nous avons repérées et de déterminer personnellement la pertinence d'une ouvre littéraire. Ensuite, je prends le temps d'expliquer la valeur de ce travail de réflexion, en exposant brièvement le rôle de la narration et de l'art comme pratiques métacognitives, voire métaéthiques. Ces considérations générales se laissent aisément illustrer par le matériau que les participants mettent sur la table, toujours riche. Nous explicitons ensemble leurs différents cadrages du texte et cherchons à comprendre ce qui sous-tend ces différences dans les trajets interprétatifs. Nous réfléchissons aux dimensions partagées, conventionnelles, de ces trajets, qui se révèlent être des habitus de lecture partagés malgré une impression de subjectivité (à laquelle,

${ }^{12}$ Voir, dans ce même numéro, la contribution de Raphaël Baroni, qui détaille bien ce mécanisme dans l'interprétation de Houellebecq; ou encore Korthals Altes 2014. 
souvent, on tient fortement : il en va de notre identité !) : nous nous lisons lisant, et en comparaison avec d'autres lecteurs. L'objectif de cet exercice n'est pas une "meilleure interprétation" du texte, même si les participants se disent toujours "enrichis" d'avoir partagé différentes lectures et contextualisations, historique, sociale, psychologique ou autre. Mais ce genre de séance offre surtout un entraînement dans la formulation verbale, le partage et l'écoute d'expériences personnelles, mais justement pas trop personnelles, car par fiction interposée, un facteur important qui ménage la pudeur des participants. En outre, cet exercice de perspective-taking, d'empathie et de positionnement personnel, oblige à accomplir une fois de plus le travail imaginatif et éthique pluri-forme qu'exige déjà de nous la lecture de textes narratifs polyphoniques. Une condition importante pour cet exercice, évidemment, est d'assurer dans le groupe une écoute et un respect mutuels. Par son contenu et par ses conditions de réalisation, cet exercice postule ainsi l'existence préalable de ces valeurs qui justement sont censées être enrichies à travers lui. Il s'agit à mon sens d'une circularité inévitable et non pas "vicieuse ", dans la mesure où elle est assumée en toute lucidité. Il n'y a pas d'enseignement ni d'entraînement qui ne repose sur un parti-pris de valeurs.

Développer l'attention à la manière dont nous déterminons des voix, dans une communication ou un texte littéraire, leur autorité et leur fiabilité, ou dont nous évaluons les rapports de force s'exprimant dans une structure énonciative, réfléchir à nos manières habituelles de faire sens, de déterminer des positions de valeur et de jugement, diversifier et augmenter nos stratégies interprétatives: tout cela pourrait former un élément substantiel dans l'entraînement aux compétences médiatiques et culturelles, au-delà de leur intérêt pour les lecteurs « littéraires».

\section{Références bibliographiques}

Aczel, R. 1998. "Hearing Voices in Narrative Texts." New Literary History, 29 (3) : 467-500.

Amossy, R. 1999. "L'éthos à la croisée des disciplines: Pragmatique, rhétorique, sociologie des champs". Dans Images de soi dans le discours, sous la direction de R. Amossy et J.-M. Adam. Lausanne : Delachaux et Niestlé : 127-154.

Angot, C. 1998a. Sujet Angot. Paris : Fayard.

Angot, C. 1998b. L'Usage de la vie. Paris : Fayard.

Bakhtine, M. M. 1970. Problèmes de la poétique de Dostoïevski. Paris : Seuil. [1929].

Banfield, A. 1982. Unspeakable Sentence: Narration and Representation in the Language of Fiction. Londres : Routledge and Kegan Paul.

Baroni, R. À paraître. "The Relevance of Irrelevance in Intriguing Narratives.” Dans Relevance and Narratology, sous la direction de K. Rennhak et M. Chihaia. Berlin : de Gruyter.

Barthes, R. 1973. Le plaisir du texte. Paris : Seuil.

Beckett, S. 1990. "Not I." Dans The Complete Dramatic Works. Londres : Faber and Faber, 373383. [1972].

\section{Arborescences}


Berger, P. L. et Luckmann, T. 1991. The Social Construction of Reality: A Treatise in the Sociology of Knowledge. Harmondsworth : Penguin Books. [1966].

Blanchot, M. 1969. "Le "il", le neutre ", dans L'entretien infini. Paris : Gallimard.

Boltanski, L. et Thévenot L. 1991. De la justification: les économies de la grandeur. Paris: Gallimard.

Booth, W. 1974. A Rhetoric of Irony. Chicago : University of Chicago Press.

Bühler, K. 1965. Die Krise der Psychologie. Stuttgart: Gustav Fischer Verlag.

Donald, M. 2006. "Arts and the Cognitive Revolution." Dans The Artful Mind: Cognitive Science and the Riddle of Human Creativity, sous la direction de M. Turner. New York : Oxford University Press : 3-20.

Fludernik, M. 1993. The Fictions of Language and the Languages of Fiction: The Linguistic Representation of Speech and Consciousness. Londres : Routledge.

Fludernik, M. 1996. Towards a "Natural" Narratology. Londres : Routledge.

Genette, G. 1972. Figures III. Paris : Seuil.

Gibson, A. 1996. Towards a Postmodern Theory of Narratology. Édinbourg : Edinburgh University Press.

Goffman, E. 1974. Frame Analysis: An Essay on the Organization of Experience. New York : Harper and Row.

Gombrich, E. H. 2002. Art and Illusion: A Study in the Psychology of Pictorial Representation. New York : Phaison. [1960].

Jongeneel, E. 2006. "Silencing the Voice in Narratology? A Synopsis." Dans Stimme(n) im Text. Narratologische Positionsbestimmungen. Sous la direction de A. Blödorn, D. Langer et M. Scheffel. Berlin et New York : De Gruyter : 9-30.

Jouve, V. 1997. La poétique du roman. Paris : SEDES.

Kearns, M. 1999. Rhetorical Narratology. Lincoln : University of Nebraska Press.

Kerbrat-Orecchioni, C. 1980. L'énonciation de la subjectivité dans le langage. Paris: Armand Colin.

Kindt, T. et Mueller, H. 2003. "Narrative Theory and/or/as Theory of Interpretation." Dans What is Narratology? Questions and Answers regarding the Status of a Theory, sous la direction de T. Kindt et K. Mueller. Berlin/New York : de Gruyter : 205-219.

Kindt, T. et Mueller, H. 2006. The Implied Author: Concept and Controversy. Berlin : Walter de Gruyter.

Koestler, A. 1989. The Act of Creation. Harmondsworth: Penguin. [1964].

Korthals Altes, E. J. 2005. "Ironie, éthos textuel et cadre de lecture : Le cas de Sujet Angot». Dans L'expérience de lecture, sous la direction de V. Jouve. Paris : Éditions L'Improviste: 85-100.

Korthals Altes, E. J. 2006. "Voice, Irony and Ethos: The Paradoxical Elusiveness of Michel Houellebecq's polemic writing in Les Particules élémentaires." Dans Stimme(n) im Text. Narratologische Positionsbestimmungen, sous la direction de A. Blödorn, D. Langer et M. Scheffel. Berlin/New York : De Gruyter : 165-194.

Korthals Altes, E. J. 2014. Ethos and Narrative Interpretation: The Negotiation of Meanings and Values in Fiction. Lincoln : University of Nebraska Press.

Langevin, F. 2014. "Genre, géographie et héritage : lecture éthique du style du narrateur dans L'annonce de Marie-Hélène Lafon ", Tangence, 105 : 91-108.

Langevin, F. 2015. Lire le narrateur: tensions de la narration hétérodiégétique dans le roman contemporain en France et au Québec. Montréal : Nota Bene.

Lanser, S. S. 1981. The Narrative Act: Point of View in Prose Fiction. Princeton : Princeton University Press.

Arborescences

Revue d'études françaises

ISSN: 1925-5357 
Levinas, E. 1983. Le temps et l'autre. Paris : Presses Universitaires de France.

Lippert, F. Sous presse. „Metaisierung als Wechsel narrativer und sozialer Frames am Beispiel von Felicitas Hoppe." Dans Geschichts(er)findungen. Felicitas Hoppe als Erzählerin zwischen Tradition und Transmoderne, sous la direction de S. Frank et J. Ilgner. Bielefeld : Transcript.

Maingueneau, D. 2004. Le discours littéraire: paratopie et scène d'énonciation. Paris : Armand Colin.

Meizoz, J. 2007. Postures littéraires : mises en scène modernes de l'auteur. Genève : Slatkine.

Minsky, M. 1977. "Frame theory." Dans Thinking: Readings in Cognitive Science, sous la direction de P.N. Johnson-Laird et P.C. Wason. Cambridge : Cambridge University Press: 355-376.

Phelan, J. 1989. Reading People, Reading Plots: Character, Progressions, and the Interpretation of Narrative. Chicago : University of Chicago Press.

Sperber, D. et D. Wilson. 1995. Relevance: Communication and Cognition. Oxford : Blackwell. [1986].

Tsur, R. 2006. Kubla Khan: Poetic Structure, Hypnotic Quality and Cognitive Style: A Study in Mental, Vocal, and Critical Performance. Amsterdam : Benjamins.

Wagner, F. 2012. "Aimez-vous "Genette" ? (Éloge de la poétique cum grano salis) ", Fabula-LhT 10, sous la direction de F. Pennanech. URL : http://www.fabula.org/lht/10/wagner.html. Page consultée le 2 octobre 2015.

Wolf, W. 2006. "Introduction: Frames, Framings and Framing Borders in Literature and Other Media." Dans Framing Borders in Literature and Other Media, sous la direction de W. Wolf et W. Bernhart. Amsterdam : Rodopi : 1-41. 